\title{
Patient-Related Risk Factors for Unplanned 30-Day Hospital Readmission Following Primary and Revision Total Knee Arthroplasty: A Systematic Review and Meta-Analysis
}

\author{
Daniel Gould ${ }^{1, *(\mathbb{D},}$, Michelle M Dowsey ${ }^{1,2}{ }^{\mathbb{D}}$, Tim Spelman ${ }^{1}$, Olivia Jo ${ }^{1}$, Wassif Kabir ${ }^{1}$, Jason Trieu ${ }^{1}{ }^{(\mathbb{D}}$, \\ James Bailey ${ }^{3}$, Samantha Bunzli ${ }^{1}$ and Peter Choong ${ }^{1}$ \\ 1 Department of Surgery, University of Melbourne, St. Vincent's Hospital Melbourne, \\ 3065 Melbourne, Australia; mmdowsey@unimelb.edu.au (M.M.D.); tim@burnet.edu.au (T.S.); \\ jo.olivia1310@gmail.com (O.J.); kabir.wassif@gmail.com (W.K.); jtrieumd@gmail.com (J.T.); \\ samantha.bunzli@unimelb.edu.au (S.B.); pchoong@unimelb.edu.au (P.C.) \\ 2 Department of Othopaedics, St. Vincent's Hospital Melbourne, 3065 Melbourne, Australia \\ 3 School of Computing and Information Systems, University of Melbourne, 3052 Melbourne, Australia; \\ baileyj@unimelb.edu.au \\ * Correspondence: Daniel.gould@unimelb.edu.au; Tel.: +61-392313955
}

Citation: Gould, D.; Dowsey, M.M; Spelman, T.; Jo, O.; Kabir, W.; Trieu, J.; Bailey, J.; Bunzli, S.; Choong, P. Patient-Related Risk Factors for Unplanned 30-Day Hospital Readmission Following Primary and Revision Total Knee Arthroplasty: A Systematic Review and Meta-Analysis. J. Clin. Med. 2021, 10, 134. https://doi.org/10.3390/ jcm10010134

Received: 4 November 2020 Accepted: 28 December 2020 Published: 2 January 2021

Publisher's Note: MDPI stays neutral with regard to jurisdictional clai$\mathrm{ms}$ in published maps and institutional affiliations.

Copyright: (C) 2021 by the authors. Licensee MDPI, Basel, Switzerland. This article is an open access article distributed under the terms and conditions of the Creative Commons Attribution (CC BY) license (https:// creativecommons.org/licenses/by/ $4.0 /)$.

\begin{abstract}
Total knee arthroplasty (TKA) is a highly effective procedure for advanced osteoarthritis of the knee. Thirty-day hospital readmission is an adverse outcome related to complications, which can be mitigated by identifying associated risk factors. We aimed to identify patient-related characteristics associated with unplanned 30-day readmission following TKA, and to determine the effect size of the association between these risk factors and unplanned 30-day readmission. We searched MEDLINE and EMBASE from inception to 8 September 2020 for English language articles. Reference lists of included articles were searched for additional literature. Patients of interest were TKA recipients (primary and revision) compared for 30-day readmission to any institution, due to any cause, based on patient risk factors; case series were excluded. Two reviewers independently extracted data and carried out critical appraisal. In-hospital complications during the index admission were the strongest risk factors for 30-day readmission in both primary and revision TKA patients, suggesting discharge planning to include closer post-discharge monitoring to prevent avoidable readmission may be warranted. Further research could determine whether closer monitoring post-discharge would prevent unplanned but avoidable readmissions. Increased comorbidity burden correlated with increased risk, as did specific comorbidities. Body mass index was not strongly correlated with readmission risk. Demographic risk factors included low socioeconomic status, but the impact of age on readmission risk was less clear. These risk factors can also be included in predictive models for 30-day readmission in TKA patients to identify high-risk patients as part of risk reduction programs.
\end{abstract}

Keywords: readmission; arthroplasty; knee; risk; patient; prognosis

\section{Introduction}

Total knee arthroplasty (TKA) is a highly effective treatment for advanced osteoarthritis of the knee [1,2]. The number of procedures being performed each year continues to grow [3]. However, despite its widespread success and increasing utilisation rates, a range of complications can arise following TKA surgery [4], some of which necessitate hospital readmission. Hospital readmission following TKA is often a marker of surgical complications [5,6]. Post-operative hospital readmissions are recognised as a significant cause for concern because such events disrupt the patient's postoperative recovery, incur significant costs to the healthcare system, and controversially have been used as a marker of quality of care [7-9]. In the USA, unplanned hospital readmissions for all patient populations overall are estimated to cost over USD 17 billion [10]. In 2004, Germany became the first country to implement financial incentives to reduce readmissions [11]. Five years later, 
in 2009, the United States' Centers for Medicare \& Medicaid Services introduced the Hospital Readmissions Reduction Program [9] and expanded this program in 2014 to include TKA $[12,13]$, where hospital penalties are applied for readmission signalling government authorities to view readmission as an issue requiring attention.

By improving our ability to accurately predict individual patients' risk of readmission following TKA, we may reduce the rate of avoidable readmissions through optimising shared decision-making and consent processes; better preparing patients for surgery and developing personalised management and discharge planning for higher risk patients. Furthermore, some readmissions are potentially avoidable and can be targeted through the development of personalised management and discharge planning for higher risk patients. Patients whose risk of readmission is difficult to mitigate, such as those patients with non-modifiable risk factors, can still benefit from risk prediction because they will be more informed as they work with the surgeon to weigh the potential benefits and risks of the procedure, and patients who have more realistic expectations regarding their likely postoperative course are more likely to achieve a satisfactory outcome following TKA [14].

Our previous narrative literature review has explored patient-related risk factors for 30-day readmissions in TKA [15]. Comorbidities such as diabetes, chronic kidney disease, and bleeding disorders were consistently shown to increase risk, but demographic factors such as age, sex, and body mass index (BMI) demonstrated a more complex and less consistent influence on 30-day readmission. Using age as an example, some researchers dichotomise at a particular number, while others create multiple categories at different cutpoints with different interval durations, such as five years or 10 years. The inconsistent way different researchers categorise predictor variables when modelling risk, and the increasing age, BMI, and physical activity demands of the TKA patient population over time [16-18], may contribute and confound this further. Thus, the purpose of this systematic review was to apply rigorous systematic review methodology to synthesise the evidence pertaining to patient risk factors for 30-day readmission following TKA. Contrasting the more subjective style of the narrative review, which identified broad themes in the literature, the current work synthesises the evidence in the most objective manner possible, including: critical appraisal of all included articles, quantitative synthesis when appropriate, narrative synthesis when quantitative synthesis was not possible, and summary of findings in accordance with a version of the Grading of Recommendations Assessment, Development and Evaluation (GRADE) approach modified for systematic reviews of prognostic factors [19].

\section{Objectives}

The objectives of this review were to (1) identify patient-related characteristics that are associated with increased risk of unplanned 30-day readmission following TKA and (2) determine the effect size of the association between the identified risk factors and unplanned 30-day readmission [20]. This systematic review and meta-analysis synthesises existing knowledge, determines the effect size of identified factors, and aims to resolve uncertainty when discrepancies arise between reports.

\section{Methods}

\subsection{Protocol and Registration}

The protocol for this systematic review has been published [20] and registered with the International Prospective Register of Systematic Reviews (PROSPERO-CRD42019118154). Protocol deviations, and justifications for them, are outlined in Supplementary File 1 (Section S1-Amendments to Protocol). Deviations were minor and did not alter the objectives, nor the direction of this systematic review. This review was conducted in accordance with the Preferred Reporting Items for Systematic Reviews and Meta-Analyses (PRISMA) statement [21]. 


\subsection{Eligibility Criteria}

We used the adapted Patient, Intervention, Comparator, Outcome (PICO) framework [22] to answer the following questions: which patient-related factors confer increased risk of unplanned 30-day readmission following TKA, and to what extent do these factors influence the risk? Eligible studies involved TKA recipients (primary and revision) compared for 30-day readmission to any institution, due to any cause, on the basis of patient risk factors (presence vs absence of each risk factor). Case series were excluded, but all other types of quantitative study design were eligible for inclusion, including retrospective and observational studies.

No restrictions were placed on the date of publication for this review.

\subsection{Information Sources, Search Strategy, and Study Inclusion}

MEDLINE and EMBASE were searched from inception to 5 February 2020, using search terms related to total knee arthroplasty and patient-related risk factors for 30-day readmission. An experienced research librarian was consulted to assist with development of the search strategy, and no restriction was placed on grey literature. The full search strategy for both databases is available in Supplementary File 1 (Section S2-Search Strategies (MEDLINE(Ovid) and EMBASE(Ovid)). The search was repeated immediately prior to final analysis (8th September 2020) to obtain studies that had been published since initially searching the database. Reference lists of articles included after full text screening were also screened.

The search strategy was carried out in line with the protocol. In brief, two reviewers (DG and OJ) pilot-tested the eligibility criteria on a 10\% sample of titles and abstracts of articles obtained from the database search. A third reviewer (MD) arbitrated discussions between DG and OJ to resolve any major difference in opinion arising from this process. The eligibility criteria were then applied to titles and abstracts and, subsequently, to the full text of articles that were deemed eligible for inclusion following initial screening.

Authors of articles potentially eligible for inclusion were contacted to provide the requisite data for inclusion.

\subsection{Data Collection Process}

Two reviewers (DG and JT) independently extracted data using a standardised data extraction form. The process was pilot-tested on 10 articles from the final set of included articles following full-text screening. Any points of clarification were resolved by discussion between DG and JT. DG identified additional information required in order to complete the critical appraisal assessment and this was verified by JT as well as the reviewer who completed the critical appraisal assessment with DG (WK).

\subsection{Data Items}

Data items extracted were those listed in the protocol, as well as several additional items necessary for critical appraisal (Supplementary File 1, Tables S3-S7).

As outlined in the protocol amendments (Supplementary File 1, Section S1), adjusted odds ratios (ORs) were preferentially collected over risk ratios (RRs), as this reflects how data were reported in the vast majority of included studies, which were retrospective in nature. As the estimated 30-day readmission rate for TKA ranges from 3\% [5] to $4.6 \%$ [23] and this is well below the $10 \%$ threshold whereby the OR provides a reasonable approximation of the RR $[20,24]$, it is unlikely that this would have altered the key findings.

\subsection{Risk of Bias of Individual Studies}

The Joanna Briggs Institute (JBI) critical appraisal tool [25] was used for all studies. A semi-quantitative method was used, based on that used by Goplen et al. [26] in a systematic review on outcomes in total joint arthroplasty (TJA) patients. However, since there is no valid cut-off value to determine "high" or "low" methodological quality, we assessed quality in relative terms by splitting the studies according to risk of bias quartiles. The item: 
"Were the groups/participants free of the outcome at the start of the study (or at the moment of exposure)?" was omitted from the checklist as it is not possible for a patient to be readmitted prior to their TKA procedure. The remaining 10 items were given equal weighting with a higher score indicating greater risk of bias. Each "no" was given a score of 2, each "unclear a score of 1 , and each "yes" a score of 0 . Arbitrarily selecting a cut-point above which a study is considered to be at high risk of bias is problematic because there are no clear guidelines pertaining to the choice of such a cut-point. Instead, quartiles of risk of bias were calculated to distinguish between higher quality studies (lower risk of bias quartiles) and lower quality studies (higher risk of bias quartiles). This use of quartiles is similar to that used by Detweiler et al., 2016 [27] in their exploration of methodological quality of systematic reviews, and we believe it provides readers with an easily interpretable way of judging the relative methodological quality of included studies without generating an overwhelming number of categories.

Selective reporting was also assessed for each study. This was included in the summary table for critical appraisal but because it was not a JBI checklist item, it did not contribute to the calculation of risk of bias score.

Table 1 depicts a generic example of the way in which the outcome of critical appraisal is presented in this review for an imaginary study, for the sake of illustration.

Table 1. Example of critical appraisal table.

\begin{tabular}{|c|c|c|c|c|c|c|c|c|c|c|c|c|}
\hline \multicolumn{13}{|c|}{ Critical Appraisal } \\
\hline $\begin{array}{l}\text { Study } \\
\text { ID }\end{array}$ & $\begin{array}{c}{ }^{*} \mathrm{JBI} \\
1 .\end{array}$ & $\begin{array}{c}\text { JBI } \\
2 .\end{array}$ & $\begin{array}{c}\text { JBI } \\
3 .\end{array}$ & $\begin{array}{c}\text { JBI } \\
4 .\end{array}$ & $\begin{array}{c}\text { JBI } \\
5 .\end{array}$ & $\begin{array}{c}\text { JBI } \\
7 .\end{array}$ & $\begin{array}{c}\text { JBI } \\
8 .\end{array}$ & $\begin{array}{c}\text { JBI } \\
9 .\end{array}$ & $\begin{array}{l}\text { JBI } \\
10 .\end{array}$ & $\begin{array}{l}\text { JBI } \\
11 .\end{array}$ & $\begin{array}{c}\text { Overall Risk of } \\
\text { Bias Score } \\
\text { (Quartile; } \\
\text { Arranged in } \\
\text { Descending Order) }\end{array}$ & $\begin{array}{l}\text { Evidence of } \\
\text { Selective } \\
\text { Reporting }\end{array}$ \\
\hline $\begin{array}{l}\text { Example } \\
\text { study }\end{array}$ & Y & Y & Y & Y & Y & Y & $\mathrm{Y}$ & $\mathrm{N}$ & $\mathrm{U}$ & $\mathrm{Y}$ & $\begin{array}{c}3 / 20 \text { therefore } 15 \% \\
\text { (1st quartile) }\end{array}$ & No \\
\hline
\end{tabular}

The colour scheme mimics that of a traffic light system indicating whether each critical appraisal criterion was fulfilled in the given study: Green $=$ yes $(\mathrm{Y})$, Yellow $=$ unclear $(\mathrm{U})$, Red $=\mathrm{No}(\mathrm{N})$; ${ }^{*}$ Joanna Briggs Institute critical appraisal checklist for cohort studies - components: Joanna Briggs Institute (JBI) 1. Were the groups similar and recruited from the same population? JBI 2. Were the exposures measured similarly to assign people to both exposed and unexposed groups? JBI 3 . Was the exposure measured in a valid and reliable way? JBI 4. Were confounding factors identified? JBI 5 . Were strategies to deal with confounding factors stated? JBI 6. (Omitted because it is not relevant) Were the groups/participants free of the outcome at the start of the study (or at the moment of exposure)? JBI 7. Were strategies to deal with confounding factors stated? JBI 8. Was the follow up time reported and sufficient to be long enough for outcomes to occur? JBI 9. Was follow up complete, and if not, were the reasons for loss to follow up described and explored? JBI 10. Were strategies to address incomplete follow up utilized? JBI 11. Was appropriate statistical analysis used?

\subsection{Risk of Bias across Studies}

Risk of bias across studies, i.e., publication bias, was minimised by using a comprehensive systematic literature search strategy and placing no restriction on grey literature.

Although articles in languages other than English were excluded, in order to determine if this created a potential source of bias, those with available English language abstracts were screened to determine the likelihood of their inclusion if the full text was available in English.

\subsection{Synthesis of Results}

Summary of findings tables were constructed, according to the modified Grading of Recommendations Assessment, Development and Evaluation (GRADE) approach [19], for each of two categories of patient-related risk factors: comorbidities and demographics. Variables that did not fit into either of these categories were summarised in a separate summary of findings table. A summary of findings table was also produced for the studies that reported on revision TKA patients (see Supplementary File 1 Table S8). For each risk factor, we considered the seven GRADE criteria [19] (study limitations, inconsistency, indirectness, imprecision, publication bias, moderate/large effect size, dose effect) in 
addition to phase of study (phase 1 = explanatory research aimed to identify associations between potential prognostic factors and the outcome; phase 2 = explanatory research aimed to confirm independent associations between potential prognostic factors and the outcome; phase 3 = research aimed to understand prognostic pathways). For each of these eight criteria, a score of one was given when there was no serious limitation identified, and a score of zero when a serious limitation was identified. A score of $<3$ was considered very low quality $=$ very little confidence in the effect estimate: true effect likely to be substantially different from the estimate of effect; a score of 3 was considered low quality $=$ confidence in the effect estimate is limited: the true effect may be substantially different from the estimate of the study; a score of 4 or 5 was considered moderate quality = moderately confident in the effect estimate: true effect is likely to be close to the estimate of the effect, but there is a possibility that it is substantially different; a score of $>5$ was considered high quality $=$ very confident that the true effect lies close to that of the estimate of the effect.

A database can be analysed in different studies, such that multiple papers included in this review report findings from analyses on the same cohort of patients, giving rise to the potential for sample dependence. In such cases, when these studies had overlapping time periods (e.g., one study analysed the 2011-2014 cohort and another the 2011-2012 cohort), the study with the longest period of data collection was selected as the representative study for that cohort, for each variable. When two or more studies had the same data collection period, the study with higher methodological quality was selected as the representative study. Although only these representative studies were included in the summary of findings tables, data from all studies are available in Supplementary File 1 (Tables S9-S13).

Table 2 depicts a generic example of the way in which the summary of findings is presented in this review for some imaginary studies and risk factors, for the sake of illustration. For the narrative synthesis, + indicates the risk factor increased risk of readmission, 0 indicates it was not correlated, and - indicates it was protective against readmission.

Table 2. Example of summary of findings table.

\begin{tabular}{|c|c|c|c|c|c|c|c|}
\hline \multicolumn{8}{|c|}{ Meta-analysis } \\
\hline Prognostic factor & \multicolumn{2}{|c|}{ OR $(95 \% \mathrm{CI})$} & \multicolumn{5}{|c|}{ Overall quality } \\
\hline Example prognostic factor 1 & \multicolumn{2}{|c|}{$0.82(0.71-0.95)$} & \multicolumn{5}{|c|}{$5(+++)$} \\
\hline \multicolumn{8}{|c|}{ Narrative Synthesis } \\
\hline \multirow{2}{*}{ Prognostic factor } & \multicolumn{2}{|c|}{ Univariate } & \multicolumn{4}{|c|}{ Multivariate } & \multirow{2}{*}{ Overall quality } \\
\hline & + & 0 & - & + & 0 & - & \\
\hline Example prognostic factor 2 & - & - & - & - & 1 & - & $4(+++)$ \\
\hline \multicolumn{8}{|c|}{ Example category 2} \\
\hline Example prognostic factor 3 & - & - & - & 2 & - & - & $5(+++)$ \\
\hline
\end{tabular}

Overall quality: +++ moderate quality = moderately confident in the effect estimate: true effect is likely to be close to the estimate of the effect, but there is a possibility that it is substantially different.

Results from studies with unadjusted effect estimates and univariate comparisons were also included, alongside additional analyses conducted in individual studies (Supplementary File 1, Tables S9-S13).

When multiple reports on a particular risk factor from the same study cohort were identified, the article with the longest follow-up period was used as the representative study for all overlapping studies with comparable eligibility criteria, but reference was made to all overlapping studies.

R statistical software [28] (version 3.5.3 (2019-03-11) -“Great Truth”) was used for meta-analysis, including the meta [29] (version 4.11-0) and tidyverse [30] (version 1.3.0) packages. The code used for meta-analysis is available in Supplementary File 1 (Section S14). 


\subsection{Sensitivity Analyses Were Conducted, Based on the Following Criteria}

1. Removal of studies with lower methodological quality, i.e., higher risk of bias.

2. Removal of studies of mixed cohorts, i.e., a combined cohort of primary and revision TKA patients.

3. Removal of studies with substantially different patient eligibility criteria to other studies, for example, those that restricted their analysis to patients over 80 years old where other studies placed no restriction on age.

If the $\mathrm{I}^{2}$ score exceeded $60 \%$ on sensitivity analysis, the variable was analysed qualitatively through narrative synthesis.

\section{Results}

\subsection{Study Selection}

Sixty-nine studies were included in this review; 374 records underwent title and abstract screening after removal of duplicates, of which 193 underwent full-text screening to include the final 69 articles, 16 of which were included in the meta-analysis. See Figure 1 for the total number of studies screened. Table 3 presents general information pertaining to all of the included studies. Supplementary File 1 (Tables S3-S7) contains detailed information on a range of variables extracted from included studies. Sixty-three articles (93\%) reported retrospective cohort studies, 57/69 (83\%) were conducted in the USA. Thirty-three of 69 studies (48\%) used the data from the American College of Surgeons' National Surgical Quality Improvement Program (NSQIP), 13/69 (19\%) used data from a single institution, and the remainder used a variety of registries and multi-hospital databases.
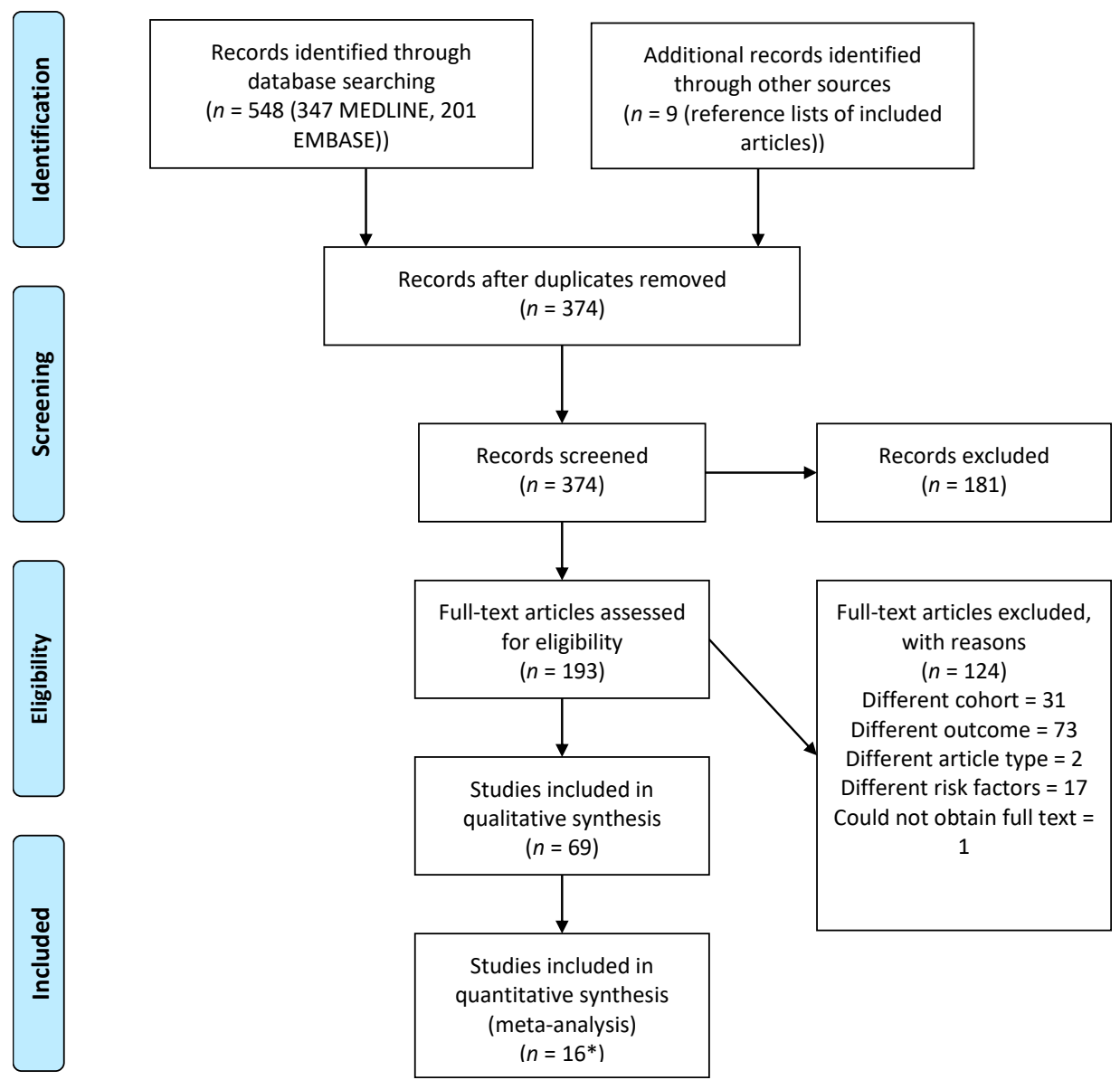

Figure 1. PRISMA Flow Diagram. PRISMA flow diagram depicting the number of articles screened and included in the review. ${ }^{*}$ included in both meta-analysis and qualitative synthesis 
Table 3. Study characteristics.

\begin{tabular}{|c|c|}
\hline Country & Study ID: Author and Year of Publication (Study Period) \\
\hline Australia & Hanly 2017 [32] (Retrospective cohort study; January 2003-December 2010) \\
\hline Canada & $\begin{array}{l}\text { Abdulla } 2020 \text { [33] (March } 2010 \text { to July 2016); Ross } 2020 \text { [34] (2003-2016); Peskun } 2012 \text { [35] } \\
\text { (1997-2007) }\end{array}$ \\
\hline Colombia & Buitrago 2020 [36] (January 1, 2012 to November 30, 2015) \\
\hline Denmark & Jorgensen 2013 [31] (1 February 2010-1 May 2011); Jorgensen 2017 [37] (2012-2014) \\
\hline Singapore & Tang 2019 [38] (January 2013 to June 2014); Tay 2017 [39] (Jan 2006-Dec 2011) \\
\hline Taiwan & Liao 2016 [40] (January 1, 2004 to December 31, 2009); Kuo 2017 [41] (January 2009 to 2012) \\
\hline UK & Ali 2019 [42] (2006-2015) \\
\hline USA & $\begin{array}{l}\text { Miric } 2014 \text { [43] (04/01/2001 to 12/31/2011); Welsh 2017 [44] (2009-2011); Kim } 2019 \text { [45] } \\
\text { (January 1,2010 to December 31, 2014); Kurtz 2016 [46] (2010-2013); Anthony } 2018 \text { [47] } \\
\text { (2013 and 2014, inclusive); Urish 2018 [48] (2014); Sodhi and Mont et al. 2019 [49] (1 } \\
\text { January } 2016 \text { to } 30 \text { September 2016); Singh 2013 [50] (2002 fiscal year); Arroyo 2019 [51] } \\
\text { (January } 2007 \text { to December } 2014 \text { for Florida and New York data, January } 2007 \text { to December } \\
2011 \text { for California data, January, } 2012 \text { to December } 2014 \text { for Maryland data); Bullock } 2003 \\
\text { [52] (January 1994-June 2000 for bilateral TKA cohort, January 1995-June } 2000 \text { for } \\
\text { unilateral TKA cohort); Charette 2019 [53] (April 2013-April 2017); D’ Apuzzo } 2017 \text { [54] } \\
\text { (1997-2014); Keeney } 2015 \text { [55] (1 January 2006-30 September 2013); Ramos } 2014 \text { [56] (2010 } \\
\text { and 2011); Ramos } 2014 \text { [56] (2010 and 2011); Ricciardi } 2017 \text { [57] (January 2010 to December } \\
\text { 2014); Saucedo 2014 [58] (2006-2010); Schaeffer 2015 [59] (July 2011-November 2012); } \\
\text { Schairer 2014 [6] (2005-2011); Workman 2019 [60] (1 June 2011-1 June 2016); Siracuse 2017 } \\
\text { [61] (2006-2011); Weick 2018 [62] (2003-2014); Kheir 2014 [63] (1 July 2009-30 June 2011); } \\
\text { Anderson 2020 [64] (2010-2014); Mudumbai 2019 [65] (2011 financial year) }\end{array}$ \\
\hline USA and various international sites & $\begin{array}{l}\text { Abola 2018 [66] (2012-2014); Alvi } 2015 \text { [67] (2005-2011); Antoniak 2020 [68] (2006-2016); } \\
\text { Belmont 2016 [69] (2011 and 2012); Bovonratwet 2018 [70] (2005-2015); Bovonratwet 2019 } \\
\text { [71] (2005-2016); Bovonratwet 2020 [72] (2012-2017); Courtney 2018 [73] (January } 12012 \\
\text { to December 31 2015); Curtis 2018 [74] (2008-2014); Curtis 2019 [75] (2012-2016); George } \\
\text { 2018 [76] (1 January 2011-31 December 2015); Gwam 2020 [77] (2008-2016); Hart 2016 [78] } \\
\text { (2011-2013); Jauregui 2015 [79] (2011); Kester 2016 [80] (January 1 2010-December } 31 \\
\text { 2013); Lehtonen 2018 [81] (2012-2015); Lovecchio 2014 [82] (2005-2011); Nowak and } \\
\text { Schemitsch 2019 [83] (2005-2016); Ottesen 2018 [84] (2005-2015); Patel } 2020 \text { [85] } \\
\text { (2011-2017); Patterson 2018 [86] (2005-2015); Pugely 2013 [23] (2011 (whole year + } 30 \text { days } \\
\text { after 31/12/11)); Robinson 2017 [87] (2012-2014; Roth } 2019 \text { [88] (unclear); Rudasill } 2019 \\
\text { [89] (2010-2016); Runner 2017 [90] (2005-2014); Sloan 2020 [91] (January 2008 to December } \\
\text { 2016); Sodhi and Anis et al. 2019 [92] (2011-2016); Suleiman 2015 [93] (2010-2012); Sutton } \\
\text { 2016 [94] (1 January 2011-31 December 2012); Webb 2017 [95] (2005-2014); Yohe 2018 [96] } \\
\text { (2008-2014); Zusmanovic 2018 [97] (1 January 2008-31 December 2015) }\end{array}$ \\
\hline
\end{tabular}

Three authors of potentially eligible articles were contacted to provide data necessary for inclusion, and one returned the required data [31].

\subsection{Risk of Bias of Individual Studies}

Table S15 (Supplementary File) shows that studies at $0-15 \%$ risk of bias (RoB) comprised the first RoB quartile, studies at $20 \%$ RoB comprised the second quartile, studies at $25-35 \%$ RoB comprised the third quartile, and studies at $40-70 \%$ RoB comprised the fourth quartile. Only $2 / 69$ studies ( $3 \%$ ) definitively addressed loss to follow-up, i.e., loss to followup occurs when patients are not routinely contacted during the 30-day post-discharge period to ascertain whether they were readmitted even if the readmission was to an institution other than that at which they underwent TKA, whereas three provided some level of justification and the remaining 64 studies clearly did not adequately address this. Similarly, only $14 / 69$ studies $(20 \%)$ clearly documented a strategy to deal with incomplete follow-up. Only $9 / 69$ studies (13\%) reported adjusted analyses with a clearly documented and robust method of handling missing data, whereas $49 / 69$ studies $(71 \%)$ reported adjusted analyses without mention of how missing data were handled, and 11/69 studies 
(16\%) reported only unadjusted analyses. These 11 studies were therefore penalised for not adequately addressing confounding results. Thirty-one studies also had evidence of selective reporting.

\subsection{Risk of Bias across Studies}

Funnel plots were deemed inappropriate due to an insufficient number $(n<10)$ of compatible studies available for meta-analysis of any risk factor [98].

The only non-English-language article eligible for title and abstract screening was in German and the abstract was available in English. Based on title and abstract screening, the article did not meet the inclusion criteria for full-text screening. Therefore, exclusion of non-English studies did not affect the findings of this review.

There was strong geographical bias with most studies being conducted in the USA, as seen in Table 3. Fifty-seven studies used data from the USA, three from Canada, two from each of Denmark, Taiwan, and Singapore, and one each from Australia, Colombia, and the UK.

In accordance with Huguet et al. 2013 [19], publication bias was assumed to be a serious limitation for every prognostic factor except those that were analysed in multiple studies of different sample sizes from relatively small ( $<10,000$ participants) to large $(>10,000$ participants). While publication bias could not be ruled out from these risk factors, we considered it unlikely to be a serious limitation when considering the overall weight of evidence for these factors. See the summary of findings tables (Tables 4-6, and Supplementary File 1, Tables S8, S16-S18) for further detail on each risk factor.

Table 4. Summary of findings-comorbidities.

\begin{tabular}{|c|c|c|}
\hline \multicolumn{3}{|c|}{ Meta-analysis } \\
\hline Prognostic factor & OR $(95 \%$ CI $)$ & Overall quality \\
\hline Alcohol abuse & $1.08(0.96-1.20)$ & $6(++++)$ \\
\hline BMI $<18.5$ vs. normal & $1.15(0.45-2.98)$ & $4(+++)$ \\
\hline BMI 25-30 vs. normal & $0.91(0.80-1.03)$ & $6(++++)$ \\
\hline BMI 30-35 vs. normal & $0.90(0.80-1.02)$ & $6(++++)$ \\
\hline BMI 35-40 vs. normal & $0.84(0.69-1.02)$ & $6(++++)$ \\
\hline BMI > 40 vs. normal & $1.05(0.84-1.31)$ & $6(++++)$ \\
\hline Obesity & $1.06(1.02-1.09)$ & $4(+++)$ \\
\hline Weight loss & $0.95(0.71-1.27)$ & $3(++)$ \\
\hline Arrhythmias and AF combined & $1.14(1.09-1.19)$ & $4(+++)$ \\
\hline $\begin{array}{l}\text { Ischaemic heart disease (IHD) + coronary artery disease (CAD) } \\
+ \text { cardiac disease }\end{array}$ & $1.29(0.79-2.09)$ & $3(++)$ \\
\hline Peripheral vascular disease & $1.17(1.10-1.24)$ & $5(+++)$ \\
\hline Previous myocardial infarction, or coronary artery disease & $1.18(0.29-4.88)$ & $3(++)$ \\
\hline Non-insulin-dependent diabetes mellitus (NIDDM) & $1.08(0.80-1.45)$ & $4(+++)$ \\
\hline Liver disease & $1.29(1.20-1.39)$ & $4(+++)$ \\
\hline Peptic ulcer disease & $0.94(0.84-1.07)$ & $4(+++)$ \\
\hline Anaemia & $1.19(1.15-1.24)$ & $6(++++)$ \\
\hline Deficiency anaemias & $1.06(1.01-1.11)$ & $4(+++)$ \\
\hline Coagulopathy & $1.25(1.15-1.36)$ & $4(+++)$ \\
\hline Fluid and electrolyte disorder & $1.05(1.00-1.12)$ & $4(+++)$ \\
\hline
\end{tabular}


Table 4. Cont.

\begin{tabular}{|c|c|c|c|c|c|c|c|}
\hline \multicolumn{8}{|c|}{ Meta-analysis } \\
\hline Chronic pulmonary disease & \multicolumn{3}{|c|}{$1.28(1.22-1.34)$} & \multicolumn{3}{|c|}{$3(++)$} & \\
\hline Paralysis & \multicolumn{3}{|c|}{$1.13(0.97-1.31)$} & \multicolumn{3}{|c|}{$4(+++)$} & \\
\hline Psychiatric disorder & \multicolumn{3}{|c|}{$1.43(1.12-1.70)$} & \multicolumn{3}{|c|}{$3(++)$} & \\
\hline Smoking & \multicolumn{3}{|c|}{$1.25(0.82-1.91)$} & \multicolumn{3}{|c|}{$5(+++)$} & \\
\hline Rheumatologic disorder & \multicolumn{3}{|c|}{$1.11(1.04-1.18)$} & \multicolumn{3}{|c|}{$3(++)$} & \\
\hline \multicolumn{8}{|c|}{ Narrative Synthesis } \\
\hline \multirow{2}{*}{ Prognostic factor } & \multicolumn{3}{|c|}{ Univariate } & \multicolumn{3}{|c|}{ Multivariate } & Overall quality \\
\hline & + & 0 & - & + & 0 & - & \\
\hline \multicolumn{8}{|c|}{ Composite comorbidity indices } \\
\hline Charlson Comorbidity Index (CCI) 1-2 (reference category =0) & - & - & - & 2 & - & - & $6(++++)$ \\
\hline CCI 1 (reference category = 0) & - & - & - & 2 & 1 & - & $6(++++)$ \\
\hline CCI 2 (reference category $=0$ ) & - & - & - & 1 & - & - & $5(++++)$ \\
\hline $\mathrm{CCI} \geq 2$ (reference category $=0$ ) & - & - & - & 1 & 1 & - & $6(++++)$ \\
\hline CCI 3-4 (reference category $=0$ ) & - & - & - & 1 & - & - & $5(+++)$ \\
\hline $\mathrm{CCI} \geq 3$ (reference category $=0$ ) & - & - & - & 1 & - & - & $4(+++)$ \\
\hline CCI 5+ (reference category $=0$ ) & - & - & - & 1 & - & - & $5(+++)$ \\
\hline Increasing CCI & - & - & - & - & 1 & - & $3(++)$ \\
\hline Presence of any comorbidity & 1 & - & - & 2 & - & - & $4(+++)$ \\
\hline $\begin{array}{l}\text { Increasing American Society of Anaesthesiologists (ASA) } \\
\text { classification (reference category = 2) }\end{array}$ & - & - & - & 1 & - & - & $4(+++)$ \\
\hline Increasing ASA classification (reference category = 1) & - & - & - & - & 1 & - & $2(+)$ \\
\hline ASA classification (other) & - & 1 & - & - & 1 & - & $2(+)$ \\
\hline Increasing Elixhauser Index & - & - & - & 1 & - & - & $5(+++)$ \\
\hline Increasing DRG (Diagnosis-related group) & - & - & - & 1 & - & - & $3(++)$ \\
\hline \multicolumn{8}{|c|}{ Cardiovascular } \\
\hline Hypertension & 2 & 2 & - & 6 & 1 & - & $5(+++)$ \\
\hline Hyperlipidaemia & 1 & 1 & - & - & - & - & $4(+++)$ \\
\hline Cardiac disease & - & - & - & 1 & - & - & $3(++)$ \\
\hline Cardiovascular disease (CVD) & - & 1 & - & - & - & - & $3(++)$ \\
\hline Congestive Cardiac/Heart Failure (CCF/CHF) & 3 & - & - & 5 & 2 & - & $5(+++)$ \\
\hline Valvular disease & 1 & - & - & 2 & 1 & - & $3(++)$ \\
\hline Peripheral vascular disease & 1 & 1 & - & - & 1 & - & $3(++)$ \\
\hline $\begin{array}{l}\text { History of percutaneous coronary intervention or cardiac } \\
\text { surgery }\end{array}$ & 1 & - & - & - & - & - & $2(+)$ \\
\hline \multicolumn{8}{|c|}{ BMI, obesity, and weight loss } \\
\hline BMI (continuous) & - & 1 & - & 1 & 1 & - & $4(+++)$ \\
\hline BMI underweight (reference category = overweight (25-30)) & - & - & - & - & 1 & - & $4(+++)$ \\
\hline BMI normal weight (reference category = overweight (25-30)) & - & - & - & - & 1 & - & $4(+++)$ \\
\hline BMI obese (reference category = overweight $(25-30)$ ) & - & - & - & - & 1 & - & $4(+++)$ \\
\hline BMI very obese (reference category = overweight (25-30)) & - & - & - & - & 1 & - & $4(+++)$ \\
\hline BMI morbidly obese (reference category = overweight (25-30)) & - & - & - & 1 & - & - & $4(+++)$ \\
\hline BMI $>30$ (reference category = normal weight) & - & - & - & 1 & - & - & $2(+)$ \\
\hline
\end{tabular}


Table 4. Cont.

\begin{tabular}{|c|c|c|c|c|c|c|c|}
\hline \multicolumn{8}{|c|}{ Meta-analysis } \\
\hline Increasing BMI (reference category $=<25$ ) & - & 1 & - & - & - & - & $1(+)$ \\
\hline Obesity & 1 & 1 & - & 2 & 1 & - & $2(+)$ \\
\hline Morbid obesity & - & 1 & - & - & - & - & $2(+)$ \\
\hline Weight loss & - & 1 & - & - & 1 & - & $4(+++)$ \\
\hline \multicolumn{8}{|c|}{ Endocrine } \\
\hline $\begin{array}{l}\text { Diabetes (general category and Peskun type two diabetes } \\
\text { mellitus (T2DM)) }\end{array}$ & - & 1 & - & 5 & 4 & - & $3(++)$ \\
\hline Diabetes (with complications) & 1 & - & - & 1 & - & - & $5(+++)$ \\
\hline Diabetes (without complications) & 1 & - & - & 1 & - & - & $5(+++)$ \\
\hline Insulin-dependent diabetes mellitus (IDDM) & 1 & - & - & 1 & - & - & $5(+++)$ \\
\hline NIDDM & 1 & - & - & - & 1 & - & $4(+++)$ \\
\hline Hypothyroidism & - & 1 & - & - & 1 & - & $5(+++)$ \\
\hline \multicolumn{8}{|c|}{ Gastrointestinal } \\
\hline Liver disease & 1 & - & - & - & 1 & - & $2(+)$ \\
\hline \multicolumn{8}{|c|}{ Haematological } \\
\hline Anaemia & - & - & - & 1 & 1 & 1 & $4(+++)$ \\
\hline Anaemia (blood loss) & 1 & - & - & - & 1 & - & $4(+++)$ \\
\hline Anaemia (deficiency) & 1 & - & - & - & 2 & - & $3(++)$ \\
\hline Bleeding disorders & 1 & - & - & 1 & - & - & $3(++)$ \\
\hline Coagulopathy & 1 & - & - & - & - & - & $3(++)$ \\
\hline Anticoagulant therapy & - & - & - & - & 1 & - & $2(+)$ \\
\hline Increasing INR & 1 & - & - & 1 & - & - & $4(+++)$ \\
\hline Fluid and electrolyte disorder & 1 & - & - & 1 & - & - & $3(++)$ \\
\hline Elevated serum blood urea nitrogen (BUN) & 1 & - & - & 1 & - & - & $4(+++)$ \\
\hline Hyponatraemia & - & 1 & - & - & 1 & - & $4(+++)$ \\
\hline Low albumin & - & - & - & 1 & - & - & $4(+++)$ \\
\hline Elevated creatinine & 1 & - & - & - & - & - & $3(++)$ \\
\hline Elevated WBC count & 1 & - & - & - & - & - & $3(++)$ \\
\hline Reduced haematocrit & 1 & - & - & - & - & - & $3(++)$ \\
\hline Low platelets & 1 & - & - & - & - & - & $3(++)$ \\
\hline \multicolumn{8}{|c|}{ Respiratory } \\
\hline $\begin{array}{l}\text { Chronic obstructive pulmonary disease (COPD) and chronic } \\
\text { airways disease (combined) }\end{array}$ & - & - & - & 4 & - & - & $3(++)$ \\
\hline Pulmonary disease & 1 & - & - & 1 & 1 & - & $4(+++)$ \\
\hline Smoking & 1 & 2 & - & 1 & - & - & $3(++)$ \\
\hline Pulmonary circulation disorder & - & - & - & 1 & 1 & - & $2(+)$ \\
\hline Asthma & - & 1 & - & - & - & - & $1(+)$ \\
\hline Dyspnoea & 1 & - & - & - & 1 & - & $3(++)$ \\
\hline Previous pneumonia & - & - & - & - & 1 & - & $3(++)$ \\
\hline Obstructive sleep apnoea & - & 1 & - & - & - & - & $1(+)$ \\
\hline Cardiopulmonary disease & 1 & - & - & - & - & - & $0(+)$ \\
\hline
\end{tabular}


Table 4. Cont.

\begin{tabular}{|c|c|c|c|c|c|c|c|}
\hline \multicolumn{8}{|c|}{ Meta-analysis } \\
\hline \multicolumn{8}{|c|}{ Psychiatric } \\
\hline Depression & 1 & 2 & - & 3 & 1 & - & $5(+++)$ \\
\hline 'Other' mental health condition (other than depression) & - & - & - & 1 & - & - & $3(++)$ \\
\hline Bipolar disorder & - & 1 & - & - & - & - & $1(+)$ \\
\hline Post-traumatic stress disorder (PTSD) & - & 1 & - & - & - & - & $1(+)$ \\
\hline Anxiety disorder & - & 2 & - & - & - & - & $2(+)$ \\
\hline Alcohol abuse & 1 & 2 & - & - & - & - & $3(++)$ \\
\hline $\begin{array}{l}\text { Drug abuse (including general substance abuse designation, } \\
\text { and drug/alcohol abuse (combined category in Kurtz)) }\end{array}$ & 1 & 1 & - & 2 & - & - & $4(+++)$ \\
\hline Psychoses & 1 & - & - & - & - & - & $2(+)$ \\
\hline \multicolumn{8}{|c|}{ Neoplastic } \\
\hline History of cancer & 1 & - & - & 2 & 1 & - & $4(+++)$ \\
\hline Disseminated cancer & 1 & - & - & 1 & 2 & - & $3(++)$ \\
\hline Lymphoma & - & - & - & 2 & - & - & $4(+++)$ \\
\hline \multicolumn{8}{|c|}{ Neurological } \\
\hline Previous stroke & - & 1 & - & 1 & - & 1 & $4(+++)$ \\
\hline Dementia & - & - & - & 1 & - & - & $4(+++)$ \\
\hline Other neurological disorder & 1 & - & - & 2 & 1 & - & $3(++)$ \\
\hline \multicolumn{8}{|c|}{ In-hospital complications } \\
\hline Deep vein thrombosis & - & - & - & 1 & - & - & $5(+++)$ \\
\hline Pulmonary embolism & - & - & - & 1 & - & - & $5(+++)$ \\
\hline Any complication & 1 & - & - & 1 & - & - & $6(++++)$ \\
\hline Any medical complication & 1 & - & - & 1 & - & - & $4(+++)$ \\
\hline Any surgical complication & 1 & - & - & 1 & - & - & $4(+++)$ \\
\hline Urinary tract infection & - & - & - & 1 & - & - & $5(+++)$ \\
\hline Surgical site infection & - & - & - & 1 & - & - & $5(+++)$ \\
\hline Sepsis & 1 & - & - & - & 1 & - & $4(+++)$ \\
\hline Cardiac (including cardiac arrest and myocardial infarction) & - & - & - & 1 & - & - & $4(+++)$ \\
\hline Pneumonia & - & - & - & 1 & - & - & $5(+++)$ \\
\hline Acute renal failure & - & - & - & 1 & - & - & $5(+++)$ \\
\hline $\begin{array}{l}\text { Cerebrovascular accident (CVA) or transient ischaemic attach } \\
\text { (TIA) }\end{array}$ & 1 & - & - & - & - & - & $3(++)$ \\
\hline \multicolumn{8}{|c|}{ Renal } \\
\hline Chronic kidney disease (CKD) & 1 & - & - & 2 & 1 & - & $5(+++)$ \\
\hline Dialysis dependence & - & - & - & 1 & - & - & $4(++)$ \\
\hline Renal failure-acute, preoperative & - & - & - & - & 1 & - & $4(++)$ \\
\hline Renal failure/disease-chronicity unspecified & 1 & - & - & 5 & - & - & $6(++++)$ \\
\hline \multicolumn{8}{|c|}{ Rheumatological and autoimmune } \\
\hline Rheumatoid arthritis/collagen vascular diseases & 1 & - & - & - & - & - & $3(++)$ \\
\hline Steroid or other immunosuppressant use for chronic condition & - & - & - & 1 & - & - & $3(++)$ \\
\hline
\end{tabular}


Table 4. Cont.

\begin{tabular}{|c|c|c|c|c|c|c|c|}
\hline \multicolumn{8}{|c|}{ Meta-analysis } \\
\hline \multicolumn{8}{|c|}{ Other } \\
\hline Preoperative opioid use & - & - & - & 2 & 1 & - & $3(++)$ \\
\hline Post-discharge opioid use & - & - & - & 1 & - & - & $1(+)$ \\
\hline Preoperative medication use (general) & - & - & - & 1 & - & - & $3(++)$ \\
\hline Preoperative medication use (analgesics) & - & - & - & & 1 & - & $1(+)$ \\
\hline Preoperative medication use (anticonvulsants) & - & - & - & 1 & - & - & $2(+)$ \\
\hline $\begin{array}{l}\text { Preoperative medication use (Serotonin-norepinephrine } \\
\text { reuptake inhibitor (SNRIs)) }\end{array}$ & - & - & - & - & 1 & - & $2(+)$ \\
\hline $\begin{array}{l}\text { Preoperative medication use (Tricyclic antidepressants } \\
\text { (TCAs)) }\end{array}$ & - & - & - & - & 1 & - & $2(+)$ \\
\hline Preoperative medication use (sedatives) & - & - & - & - & 1 & - & $2(+)$ \\
\hline Wound class & 1 & - & - & - & 2 & - & $4(+++)$ \\
\hline
\end{tabular}

Overall quality: + very low quality = very little confidence in the effect estimate: true effect likely to be substantially different from the estimate of effect; ++ low quality = confidence in the effect estimate is limited: the true effect may be substantially different from the estimate of the study; +++ moderate quality = moderately confident in the effect estimate: true effect is likely to be close to the estimate of the effect, but there is a possibility that it is substantially different; ++++ high quality = very confident that the true effect lies close to that of the estimate of the effect.

Table 5. Summary of Findings-Demographics.

\begin{tabular}{|c|c|c|c|c|c|c|c|}
\hline \multicolumn{8}{|c|}{ Meta-analysis } \\
\hline Prognostic factor & \multicolumn{3}{|c|}{ OR $(95 \% \mathrm{CI})$} & \multicolumn{4}{|c|}{ Overall quality } \\
\hline Hispanic race & \multicolumn{3}{|c|}{$0.92(0.68-1.25)$} & \multicolumn{4}{|c|}{$5(+++)$} \\
\hline \multicolumn{8}{|c|}{ Narrative Synthesis } \\
\hline \multirow{2}{*}{ Prognostic factor } & \multicolumn{3}{|c|}{ Univariate } & \multicolumn{3}{|c|}{ Multivariate } & \multirow{2}{*}{$\begin{array}{l}\text { Overall } \\
\text { quality }\end{array}$} \\
\hline & + & 0 & - & + & 0 & - & \\
\hline \multicolumn{8}{|c|}{ Age (continuous variable) } \\
\hline Age & 3 & 1 & - & 4 & 3 & - & $5(+++)$ \\
\hline \multicolumn{8}{|c|}{ Sex } \\
\hline Female sex & - & 1 & - & - & 1 & 4 & $5(+++)$ \\
\hline Male sex & 2 & 2 & - & 8 & - & - & $6(++++)$ \\
\hline \multicolumn{8}{|c|}{ Race } \\
\hline Black (reference = white or non-Black) & 1 & 1 & - & 4 & 3 & - & $4(+++)$ \\
\hline Hispanic (reference = white or non-Hispanic) & - & 1 & - & - & - & - & $1(+)$ \\
\hline Asian (reference = white) & - & 2 & - & - & 1 & 2 & $4(+++)$ \\
\hline Native Hawaiian (reference = white) & - & 1 & - & - & - & - & $3(++)$ \\
\hline American Indian (reference = white) & - & 2 & - & - & 1 & - & $4(+++)$ \\
\hline White & - & 1 & 1 & - & 1 & - & $2(+)$ \\
\hline Indian (reference = Chinese) & - & - & - & - & 1 & - & $2(+)$ \\
\hline Malay (reference = Chinese) & - & - & - & - & 1 & - & $2(+)$ \\
\hline Biracial (Workman) or mixed race (Ali) & 1 & - & - & 1 & - & - & $5(+++)$ \\
\hline Minority ethnicity & - & - & - & 1 & - & - & $2(+)$ \\
\hline Other $($ Tang $=$ Chinese; otherwise $=$ white $)$ & - & 1 & - & 1 & 4 & 2 & $3(++)$ \\
\hline
\end{tabular}


Table 5. Cont.

\begin{tabular}{|c|c|c|c|c|c|c|c|}
\hline \multicolumn{8}{|c|}{ Meta-analysis } \\
\hline Missing & - & 1 & - & 1 & - & 3 & $3(++)$ \\
\hline $\begin{array}{l}\text { Race (combined analysis - i.e., racial difference exists } \\
\text { between readmitted and non-readmitted cohorts) }\end{array}$ & 2 & - & - & 1 & - & - & $4(+++)$ \\
\hline \multicolumn{8}{|c|}{ Socioeconomic } \\
\hline Decreasing incoming & - & - & - & 3 & 1 & - & $5(+++)$ \\
\hline Low socioeconomic status & 1 & - & - & 1 & - & - & $4(+++)$ \\
\hline \multicolumn{8}{|c|}{ Insurance status } \\
\hline $\begin{array}{c}\text { Medicare (reference category = private insurance or } \\
\text { non-Medicare) }\end{array}$ & - & - & - & 4 & - & - & $4(+++)$ \\
\hline Medicaid (reference category = private insurance) & - & - & - & 3 & - & - & $3(++)$ \\
\hline $\begin{array}{l}\text { Self-pay, no charge, workers' compensation, or other } \\
\text { (reference category = private insurance) }\end{array}$ & - & - & - & - & 2 & 3 & $2(+)$ \\
\hline Disability entitlement & - & - & - & 1 & - & - & $3(++)$ \\
\hline \multicolumn{8}{|c|}{ Functional status, living situation, and frailty } \\
\hline Dependent functional status & - & - & - & 1 & - & - & $4(+++)$ \\
\hline Use of walking aids & 1 & - & - & - & 1 & - & $4(+++)$ \\
\hline Living alone & - & 1 & - & - & 2 & - & $5(+++)$ \\
\hline Living in an institution or nursing home & - & 1 & - & 1 & - & - & $3(++)$ \\
\hline Homeless & - & - & - & 1 & - & - & $2(+)$ \\
\hline Frailty (Modified Frailty Index) & - & - & - & 1 & - & - & $5(+++)$ \\
\hline
\end{tabular}

Overall quality: + very low quality = very little confidence in the effect estimate: true effect likely to be substantially different from the estimate of effect; ++ low quality $=$ confidence in the effect estimate is limited: the true effect may be substantially different from the estimate of the study; +++ moderate quality = moderately confident in the effect estimate: true effect is likely to be close to the estimate of the effect, but there is a possibility that it is substantially different; ++++ high quality = very confident that the true effect lies close to that of the estimate of the effect.

\subsection{Synthesis of Evidence}

Tables 4-6, and Supplementary File 1 (Tables S8, S16-S18), present the findings for every risk factor identified in the included studies, including high, moderate, low, and very low-quality evidence. A summary of the high and moderate quality evidence for risk factors that correlated with readmission are presented. Forest plots for all meta-analyses are available (Supplementary File 1, Section S19).

\subsubsection{Comorbidities}

On meta-analysis, risk factors associated with 30-day readmission were: arrhythmias (including atrial fibrillation), anaemia, deficiency anaemias, peripheral vascular disease, liver disease, and coagulopathy. On narrative synthesis, risk factors associated with 30-day readmission were: hypertension, congestive heart failure (CHF), diabetes, elevated preoperative international normalised ratio (INR), elevated serum blood urea nitrogen (BUN), reduced serum albumin, depression, drug abuse, history of cancer, and chronic kidney disease (CKD). On narrative synthesis, increasing comorbidity burden as indicated by Charlson Comorbidity Index, Elishauser Index, and Diagnosis-Related Group roughly correlated with increased readmission risk. This relationship was not observed strongly for American College of Anaesthesiologists (ASA) Classification.

Body mass index (BMI) was categorised in different ways in various studies, but on meta-analysis none of these categories were correlated with readmission. Similarly, when BMI was presented as a continuous variable it did not consistently correlate with 
readmission. However, when presented in categories, both obesity and morbid obesity did correlate with readmission both on meta-analysis and on narrative synthesis. Note that obesity categories were not clearly defined in these studies which used the labels 'obesity' or 'morbid obesity'. It is possible that these terms were defined in line with the definitions given by the World Health Organization [99], but this was not stated.

Table 6. Summary of Findings-Other.

\begin{tabular}{|c|c|c|c|c|c|c|c|}
\hline \multicolumn{8}{|c|}{ Meta-analysis } \\
\hline Prognostic factor & \multicolumn{3}{|c|}{ OR $(95 \% \mathrm{CI})$} & \multicolumn{4}{|c|}{ Overall quality } \\
\hline Elective vs non-elective & \multicolumn{3}{|c|}{$0.82(0.71-0.95)$} & \multicolumn{4}{|c|}{$5(+++)$} \\
\hline \multicolumn{8}{|c|}{ Narrative Synthesis } \\
\hline \multirow{2}{*}{ Prognostic factor } & \multicolumn{3}{|c|}{ Univariate } & \multicolumn{3}{|c|}{ Multivariate } & \multirow{2}{*}{ Overall quality } \\
\hline & + & 0 & - & + & 0 & - & \\
\hline \multicolumn{8}{|c|}{ Operative variables } \\
\hline Elective or non-elective procedure & - & - & - & - & 1 & - & $4(+++)$ \\
\hline Emergency procedure & - & - & - & - & 1 & - & $4(+++)$ \\
\hline Traumatic indication for TKA & - & - & - & 1 & 1 & - & $4(+++)$ \\
\hline Bilateral procedure & - & 1 & - & 2 & 2 & - & $3(++)$ \\
\hline Revision surgery (vs primary) & 1 & 1 & - & 1 & - & - & $3(++)$ \\
\hline \multicolumn{8}{|c|}{ Healthcare utilisation } \\
\hline Increasing number of previous admissions & - & - & - & 2 & - & - & $5(+++)$ \\
\hline Number of prior knee procedures & - & - & - & - & - & 1 & $4(+++)$ \\
\hline GP visit between surgery and readmission & 1 & - & - & - & - & 1 & $3(++)$ \\
\hline Radiation therapy within 90 days prior to procedure & - & - & - & - & - & - & $\mathrm{N} / \mathrm{A}$ \\
\hline Chemotherapy within 30 days prior to procedure & - & 1 & - & - & - & - & $2(+)$ \\
\hline Prior operation & - & - & - & - & 1 & - & $1(+)$ \\
\hline$>30$ outpatient visits in the 365 days prior to procedure & - & - & - & 1 & - & - & $1(+)$ \\
\hline Patient-reported outcome measures & - & - & - & 1 & - & - & $1(+)$ \\
\hline Patient location & - & - & - & - & 1 & 1 & $2(+)$ \\
\hline
\end{tabular}

$\mathrm{N} / \mathrm{A}$ = not-assessable; Overall quality: + very low quality = very little confidence in the effect estimate: true effect likely to be substantially different from the estimate of effect; ++ low quality $=$ confidence in the effect estimate is limited: the true effect may be substantially different from the estimate of the study; +++ moderate quality = moderately confident in the effect estimate: true effect is likely to be close to the estimate of the effect, but there is a possibility that it is substantially different.

\subsubsection{Demographics}

Age was categorised in many ways, making a direct comparison of studies for the Summary of Findings table impractical. The findings of individual studies are all documented in Supplementary File 1 (Tables S9-S13). When age was analysed as a continuous variable three of seven studies found that older age correlated with readmission risk, one study found that younger age correlated with increased risk and the remaining three studies found no correlation.

Risk factors correlated with increased readmission risk were: male sex, Black race, decreasing income, low socioeconomic status, Medicare insurance (US-based cohort, private insurance as the reference category), dependent functional status, and frailty. Asian race was protective against readmission.

\subsubsection{Other}

Patients who underwent elective surgery were less likely to be readmitted than those who underwent urgent surgery, providing the best-available estimation of the impact of 
the urgency of the patient's condition on readmission risk. A higher number of previous hospital admissions was correlated with increased readmission risk, whereas a higher number of prior knee procedures was protective against readmission.

The following in-hospital complications that occurred during the index admission, were strongly correlated with readmission risk: any complication (combined category), any medical complication, any surgical complication, urinary tract infection (UTI), surgical site infection (SSI), cardiac complication, pneumonia, acute renal failure. The correlation between in-hospital complication and subsequent risk of 30-day readmission was stronger than for any other variable.

\subsubsection{Revision-Only Cohorts}

Meta-analysis of 30-day readmission risk for revision TKA was not possible therefore we conducted a narrative synthesis (see Supplementary File 1, Table S8). Similar to that of the primary TKA studies, in-hospital complications were strongly correlated with readmission after discharge after revision TKA. Of interest, Belmont et al. 2016 [69] and Courtney et al. 2018 [73] reported contrasting findings with respect to the impact of sex on readmission risk, with the former reporting increased risk due to female sex and the latter reporting increased risk due to male sex. Both studies analysed the NSQIP cohort, with Courtney analysing the data from 2012-2016 and Belmont analysing the data from 2011-2012. Unique to this population, revision for infectious aetiology correlated with higher rates of readmission than revision for other indications.

\subsubsection{Different Types of Readmission}

The focus of this review is on 30-day readmission due to any cause, so the main summary of findings tables comprise studies that analysed all-cause readmission. Three of these studies additionally analysed readmission due to specific causes: D'Apuzzo et al. 2017 [54], Ali et al. 2019 [42], and Rudasil et al. 2019 [89]. An in-depth discussion of the differences between risk factor profiles for each of these outcomes is given in the full text articles. One other study, Anthony et al. 2018 [47], did not analyse all-cause readmission, instead focusing on readmission due to surgical site infection only. On multivariate logistic regression analysis (OR (95\% confidence interval)), risk factors found to be associated with risk of readmission were: age 18-30 (reference category <18: $0.114(0.02-0.63)$ ), female sex $(0.559(0.53-0.59))$, private insurance and Medicaid (reference category Medicare: $0.679(0.63-0.73)$ and 1.489 (1.32-1.68), respectively), hypertension (1.189 (1.11-1.27)), obesity (1.182 (1.11-1.26)), and diabetes (1.122 (1.05-1.2)). The results for each of these studies are available in Supplementary File 1 (Tables S12 and S13).

\section{Discussion}

The aims of this systematic review were to (1) identify patient-related characteristics that confer increased risk of unplanned 30-day readmission following TKA and (2) determine the effect size of the association between the identified risk factors and unplanned 30-day readmission.

The risk of readmission within 30 days of TKA is increased in the presence of a broad range of modifiable and non-modifiable patient-related risk factors in the domains of comorbidity, demographics, and socioeconomic status. In relation to the narrative review on this topic [15], the substantial impact of in-hospital complications on readmission risk is a novel finding and the evidence pertaining to the impact of age, BMI, and socioeconomic status was explored in much greater detail. Many of the risk factors analysed comprise patient information routinely collected as part of the work-up patients receive prior to TKA surgery. The findings of this review can therefore be readily used in clinically applicable predictive modelling for 30-day readmission in TKA patients.

In-hospital complications were most strongly associated with 30-day all-cause readmission, for both primary and revision TKA patients. It was beyond the scope of this study to uncover mechanistic prognostic pathways by which these risk factors influence a 
patient's likelihood of being readmitted; however, it makes intuitive sense that patients who are susceptible to in-hospital complications prior to discharge may be more susceptible than other patients to complications post-discharge, which lead to hospital readmission. This systematic review presents evidence that in-hospital complications specifically increase the risk of readmission following discharge. This finding was consistent for the broad categories of any complication, any surgical complication, and any medical complication, and also for some specific complications. These included deep vein thrombosis (DVT), pulmonary embolism (PE), urinary tract infection (UTI), surgical site infection (SSI), cardiac complications (including cardiac arrest and myocardial infarction), pneumonia, and acute renal failure. Care is already taken to prevent such complications, for example with the use of prophylactic antibiotics and venous thromboembolism prophylaxis, and patients who experience such complications prior to discharge are already managed with the appropriate measures. These measures include infection control and antimicrobial therapy, venous thromboembolism treatment, renal replacement therapy for acute renal failure, and cardiac interventions and monitoring of cardiac complications. This review provides further evidence that, even when such appropriate measures are taken to prevent and manage these complications, there is still an increased risk of readmission following discharge. As such, even after apparent stabilisation of the patient to such an extent that they are cleared for discharge, it may be prudent to continue monitoring these patients in the post-discharge period to prevent avoidable readmission. This could take the form of a scheduled outpatient or telehealth appointment within the weeks following discharge in order to check in with the patient and assess whether they have any concerns or issues that need to be addressed in order prevent readmission. Further research is required to elucidate the causes and timing of readmission post-TKA such that post-discharge monitoring for patients with in-hospital complications is allocated appropriately and delivered at the appropriate time.

\section{Strengths and Limitations}

The strengths of this study include the comprehensive search strategy and rigorous narrative synthesis and meta-analysis of an extensive range of risk factors, according to a pre-defined and published protocol [20]. Meta-analyses consistently included 100,000 to one million patients, and narrative syntheses of some variables exceeded one million patients. Heterogeneity (measured by the $\mathrm{I}^{2}$ statistic) was low in most cases. The findings were synthesised and summarised in accordance with a formulation of the GRADE framework specifically modified to suit prognostic factor systematic reviews.

Age, and to a lesser extent BMI, was categorised in many different ways, as has been previously reported [15], making comparison between studies difficult. There is no universal consensus for categorising age. The best available guidelines [100] recommend categorising age into under $1 \mathrm{y}$; single-year for 1-24 y; 5-year groupings for 25-54 y; single-year for 55-74 y; 5 year groupings for 75-85; $85 \mathrm{y}+$. No study in this review adhered to this highest level of detail. Moving forward, we recommend researchers consider utilising this categorisation scheme wherever possible to ensure optimal statistical comparisons of findings between studies.

While we did not place a restriction on the inclusion of prospective studies, 63 of the 69 included studies were retrospective cohort studies. Retrospective studies do not provide the same level of evidence as prospective studies designed specifically to analyse risk factors for readmission. Another issue was that of sample dependence, with 33 of 69 studies included in this review drawing data from the American College of Surgeons National Surgical Quality Improvement Program (NSQIP) database [101]. This posed a challenge for our analysis because studies with overlapping data collection periods needed to be treated as if they were the same study population.

Some studies reported analyses that combined both urgent and elective cases. For example, the comparison of elective TKA with non-elective TKA depicted in Table 6 is arguably flawed as these two patient populations are fundamentally different. These pa- 
tient populations were not presented separately in the included studies and therefore could not be analysed independently.

Another potential limitation of this review is the lack of account for policy changes aimed at reducing 30-day readmission rates in many of the included studies. The Hospital Readmissions Reduction Program (HRRP) in the USA was expanded to include TKA from 2015 onwards, the UK has had a national readmission reduction program in place since 2011, and Denmark has included public reporting of readmission rates and provided indirect incentives to reduce readmission rates since 2006 [11,102]. In this review, one USA study used data entirely from 2016, 38 USA studies analysed data prior to 2015, and the remaining 18 USA studies ( $18 / 57$ or $32 \%$ of all USA studies) used a data collection window that included 2015 (see Table 3). Only two of these 18 studies adjusted their analyses for year of surgery $[89,92]$, and thus providing some level of control for potential confounding introduced by the expansion of the HRRP program to include TKA in 2015. The remaining 16 studies are potentially limited by the fact that there was no consideration in the analysis for the impact of such a major policy change being introduced during their data collection period. There was only one study from a UK population (see Table 3 ), which similarly did not adjust for year of surgery nor otherwise account for the fact that the national readmission study was introduced during their data collection period. The two studies from Denmark included in this review analysed data collected after 2006 (see Table 3), the year public reporting of readmission rates commenced in Denmark. To strengthen the evidence for the risk factors included in this review, we call for future prospective cohort studies involving a more diverse range of databases from different geographical locations, taking into account major policy changes in their analyses.

\section{Conclusions}

Thirty-day all-cause readmission risk following TKA is increased in the presence of a broad range of modifiable and non-modifiable patient-related risk factors in the domains of comorbidity, demographics, and low socioeconomic status. The strongest risk factors are in-hospital complications prior to discharge, suggesting patients who suffer a complication could benefit from closer monitoring in the post-discharge period aimed at preventing avoidable readmission. Future research could be conducted to determine whether closer post-discharge monitoring would prevent unplanned but avoidable readmissions. The effects of age and BMI were difficult to analyse due to variations in the categorisation of these variables. Body mass index was not strongly correlated with readmission, while the correlation between older age and readmission was inconsistent. These findings can be used by clinicians when working with patients to reduce their risk of post-TKA readmission. Statisticians can also use these findings in predictive modelling to implement readmission risk reduction systems. A predictive model that utilises sophisticated statistical and machine learning methodologies could draw together the extensive range of variables correlated with readmission that we have identified and harness the underlying complexities of the data to make robust predictions for individual patients in order to personalise care.

Supplementary Materials: The following are available online at https:/ / www.mdpi.com/2077-038 3/10/1/134/s1, Section S1 Amendments to Protocol; Section S2 Search Strategies (MEDLINE(Ovid) and EMBASE(Ovid)); Table S3 Study cohort, geographical location, and type of study; Table S4 Study inclusion and exclusion criteria, sample size, and patient characteristics; Table S5 Outcome definition, missing data, and loss to follow-up; Table S6 Prognostic factor selection; Table S7 Adjustment for confounding; Table S8 Summary of Findings: Revision-only studies; Table S9 Results for all-cause readmission, adjusted analysis; Table S10 Results for all-cause readmission, unadjusted and secondary analyses; Table S11 Arroyo 2019 - Results from Exploratory Stratified Analysis; Table S12 Results for other types of readmission, adjusted analysis; Table S13 Results for other types of readmission, unadjusted and secondary analyses; Section S14 Meta-analysis R code; Table S15 Critical Appraisal; Table S16 Summary of Findings - Comorbidities; Table S17 Summary of Findings - Demographics; Table S18 Summary of Findings - Other; Section S19 forest plots. 
Author Contributions: D.G. drafted the manuscript, with P.C., M.M.D., T.S., J.B., S.B., O.J., W.K., and J.T. providing intellectual content. P.C., M.M.D., and T.S. co-designed the study with D.G. J.B. contributed the acquisition and analysis of data. D.G. and O.J. conducted the systematic literature search and screening. D.G. and W.K. conducted critical appraisal. D.G. and J.T. extracted data from included articles. D.G. and T.S. carried out the statistical analysis. P.C., M.M.D., T.S., J.B., S.B., O.J., W.K., and J.T. have reviewed and approved the final manuscript, and all agree to be accountable for all aspects of the manuscript and will work together to ensure questions relating to the accuracy and integrity of any part of it are appropriately investigated and resolved. All authors have read and agreed to the published version of the manuscript.

Funding: This work is supported by the National Health and Medical Research Council of Australia (NHMRC) Centre for Research Excellence in Total Joint Replacement (APP1116235). MD holds an NHMRC Career Development Fellowship (APP1122526) and a University of Melbourne Dame Kate Campbell Fellowship. PC holds an NHMRC Practitioner Fellowship (APP1154203).

Institutional Review Board Statement: Not applicable.

Informed Consent Statement: Not applicable.

Data Availability Statement: Data is contained within the article or supplementary material.

Acknowledgments: We acknowledge Anna Lovang, librarian at Carl de Gruchy Library, St Vincent's Hospital, Melbourne, for her assistance in developing the search strategies.

Conflicts of Interest: The authors declare no conflict of interest.

\section{References}

1. Ethgen, O.; Bruyere, O.; Richy, F.; Dardennes, C.; Reginster, J.Y. Health-related quality of life in total hip and total knee arthroplasty. A qualitative and systematic review of the literature. J. Bone Joint Surg. Am. Vol. 2004, 86-a, 963-974. [CrossRef] [PubMed]

2. Association, A.O. Annual Report 2017; Australian Orthopaedic Association - South Australian Health and Medical Research Institute: Adelaide, Australia, 2017.

3. Kurtz, S.; Ong, K.; Lau, E.; Mowat, F.; Halpern, M. Projections of primary and revision hip and knee arthroplasty in the United States from 2005 to 2030. J. Bone Joint Surg. Am. Vol. 2007, 89, 780-785. [CrossRef]

4. Rucco, V.; Zanetti, R.; Colonnello, V. Complications after total knee arthroplasty. Eur. J. Phys. Rehabil. Med. 2001, $37,115$.

5. Ramkumar, P.N.; Chu, C.; Harris, J.; Athiviraham, A.; Harrington, M.; White, D.; Berger, D.; Naik, A.; Li, L. Causes and rates of unplanned readmissions after elective primary total joint arthroplasty: A systematic review and meta-analysis. Am. J. Orthop. 2015, 44, 397-405. [PubMed]

6. Schairer, W.W.; Vail, T.P.; Bozic, K.J. What are the rates and causes of hospital readmission after total knee arthroplasty? Knee. Clin. Orthop. Relat. Res. 2014, 472, 181-187. [CrossRef]

7. Fischer, C.; Lingsma, H.F.; Marang-van de Mheen, P.J.; Kringos, D.S.; Klazinga, N.S.; Steyerberg, E.W. Is the readmission rate a valid quality indicator? A review of the evidence. PLOS ONE 2014, 9, e112282. [CrossRef]

8. Joynt, K.E.; Jha, A.K. Thirty-day readmissions-truth and consequences. New Engl. J. Med. 2012, 366, 1366-1369. [CrossRef]

9. Fonarow, G.C. Unintended Harm Associated With the Hospital Readmissions Reduction Program. Jama 2018, 320, $2539-2541$. [CrossRef]

10. Hamar, G.B.; Coberley, C.; Pope, J.E.; Cottrill, A.; Verrall, S.; Larkin, S.; Rula, E.Y. Effect of post-hospital discharge telephonic intervention on hospital readmissions in a privately insured population in Australia. Aust. Health Rev. 2018, 42, 241-247. [CrossRef]

11. ACSQHC. Avoidable Hospital Readmissions: Report on Australian and International Indicators, Their Use and the Efficacy of Interventions to Reduce Readmissions; Australian Commission on Safety and Quality in Health Care: Sydney, Australia, 2019.

12. McIlvennan, C.K.; Eapen, Z.J.; Allen, L.A. Hospital readmissions reduction program. Circulation 2015, 131, 1796-1803. [CrossRef]

13. Ramaswamy, A.; Marchese, M.; Cole, A.P.; Harmouch, S.; Friedlander, D.; Weissman, J.S.; Lipsitz, S.R.; Haider, A.H.; Kibel, A.S.; Schoenfeld, A.J.; et al. Comparison of Hospital Readmission After Total Hip and Total Knee Arthroplasty vs Spinal Surgery After Implementation of the Hospital Readmissions Reduction Program. JAMA Network Open 2019, 2, e194634. [CrossRef] [PubMed]

14. Tilbury, C.; Haanstra, T.M.; Leichtenberg, C.S.; Verdegaal, S.H.; Ostelo, R.W.; de Vet, H.C.; Nelissen, R.G.; Vliet Vlieland, T.P. Unfulfilled Expectations After Total Hip and Knee Arthroplasty Surgery: There Is a Need for Better Preoperative Patient Information and Education. J. Arthroplast. 2016, 31, 2139-2145. [CrossRef] [PubMed]

15. Gould, D.; Dowsey, M.; Jo, I.; Choong, P. Patient-related risk factors for unplanned 30-day readmission following total knee arthroplasty: A narrative literature review. ANZ J. Surg. 2020, 22, 22. [CrossRef] [PubMed]

16. Culliford, D.; Maskell, J.; Judge, A.; Cooper, C.; Prieto-Alhambra, D.; Arden, N.K. Future projections of total hip and knee arthroplasty in the UK: Results from the UK Clinical Practice Research Datalink. Osteoarthr. Cartil. 2015, 23, 594-600. [CrossRef] 
17. Ravi, B.; Croxford, R.; Reichmann, W.M.; Losina, E.; Katz, J.N.; Hawker, G.A. The changing demographics of total joint arthroplasty recipients in the United States and Ontario from 2001 to 2007. Best Pract. Res. Clin. Rheumatol. 2012, 26, 637-647. [CrossRef]

18. Goudie, E.B.; Robinson, C.; Walmsley, P.; Brenkel, I. Changing trends in total knee replacement. Eur. J. Orthop. Surg. Traumatol. 2017, 27, 539-544. [CrossRef]

19. Huguet, A.; Hayden, J.A.; Stinson, J.; McGrath, P.J.; Chambers, C.T.; Tougas, M.E.; Wozney, L. Judging the quality of evidence in reviews of prognostic factor research: Adapting the GRADE framework. Syst. Rev. 2013, 2, 71. [CrossRef]

20. Gould, D.; Dowsey, M.; Spelman, T.; Jo, I.; Kabir, W.; Trieu, J.; Choong, P. Patient-related risk factors for unplanned 30-day readmission following total knee arthroplasty: A protocol for a systematic review and meta-analysis. Syst. Rev. $2019,8,215$. [CrossRef]

21. Moher, D.; Liberati, A.; Tetzlaff, J.; Altman, D.G.; Group, P. Preferred reporting items for systematic reviews and meta-analyses: The PRISMA statement. PLoS Med. 2009, 6, e1000097. [CrossRef]

22. Schardt, C.; Adams, M.B.; Owens, T.; Keitz, S.; Fontelo, P. Utilization of the PICO framework to improve searching PubMed for clinical questions. BMC Med. Inform. Decis. Mak. 2007, 7, 16. [CrossRef]

23. Pugely, A.J.; Callaghan, J.J.; Martin, C.T.; Cram, P.; Gao, Y. Incidence of and risk factors for 30-day readmission following elective primary total joint arthroplasty: Analysis from the ACS-NSQIP. J. Arthroplast. 2013, 28, 1499-1504. [CrossRef]

24. Viera, A. Odds ratios and risk ratios: what's the difference and why does it matter? South. Med. J. 2008, 101, 730-734. [CrossRef] [PubMed]

25. Moola, S.; MZTufanaru, C.; Aromataris, E.; Sears, K.; Sfetcu, R.; Currie, M.; Lisy, K.; Qureshi, R.; Mattis, P.; Mu, P. Chapter 7: Systematic reviews of etiology and risk. In Joanna Briggs Institute Reviewer's Manual; The Joanna Briggs Institute: Adelaide, Australia, 2017.

26. Goplen, C.M.; Verbeek, W.; Kang, S.H.; Jones, C.A.; Voaklander, D.C.; Churchill, T.A.; Beaupre, L.A. Preoperative opioid use is associated with worse patient outcomes after total joint arthroplasty: A systematic review and meta-analysis. BMC Musculoskelet. Disord. 2019, 20, 234. [CrossRef] [PubMed]

27. Detweiler, B.; Kollmorgen, L.; Umberham, B.; Hedin, R.; Vassar, B. Risk of bias and methodological appraisal practices in systematic reviews published in anaesthetic journals: A meta-epidemiological study. Anaesthesia 2016, 71, 955-968. [CrossRef] [PubMed]

28. Team, R.C. R: A Language and Environment for Statistical Computing; R Foundation for Statistical Computing: Vienna, Austria, 2013.

29. Schwarzer, G. meta: An R package for meta-analysis. R News 2007, 7, 40-45.

30. Wickham, H.; Wickham, M.H. Package tidyverse. In Easily Install and Load the 'Tidyverse; World Health Organization: Geneva, Switzerland, 2017.

31. Jorgensen, C.C.; Kehlet, H.; Lundbeck Foundation Centre for Fast-track, H.; Knee Replacement Collaborative, G. Outcomes in smokers and alcohol users after fast-track hip and knee arthroplasty. Acta Anaesthesiol. Scand. 2013, 57, 631-638. [CrossRef]

32. Hanly, R.J.; Marvi, S.K.; Whitehouse, S.L.; Crawford, R.W. Morbid Obesity in Total Knee Arthroplasty: Joint-Specific Variance in Outcomes for Operative Time, Length of Stay, and Readmission. J. Arthroplast. 2017, 32, 2712-2716. [CrossRef]

33. Abdulla, I.; Mahdavi, S.; Khong, H.; Gill, R.; Powell, J.; Johnston, K.D.; Sharma, R. Does body mass index affect the rate of adverse outcomes in total hip and knee arthroplasty? A retrospective review of a total joint replacement database. Can. J. Surg. 2020, 63, E142-E149. [CrossRef]

34. Ross, T.D.; Dvorani, E.; Saskin, R.; Khoshbin, A.; Atrey, A.; Ward, S.E. Temporal Trends and Predictors of Thirty-Day Readmissions and Emergency Department Visits Following Total Knee Arthroplasty in Ontario Between 2003 and 2016. J. Arthroplast. 2020, 35, 364-370. [CrossRef]

35. Peskun, C.; Mayne, I.; Malempati, H.; Kosashvili, Y.; Gross, A.; Backstein, D. Cardiovascular disease predicts complications following bilateral total knee arthroplasty under a single anesthetic. Knee 2012, 19, 580-584. [CrossRef]

36. Buitrago, G.; Ortiz, J.R.; Camacho, F. Clinical Outcomes, Health Care Costs and Prognostic Factors for Total Knee Arthroplasty: A Multilevel Analysis of a National Cohort Study Using Administrative Claims Data. J. Knee Surg. 2020, 24, 24. [CrossRef] [PubMed]

37. Jørgensen, C.C.; Pitter, F.T.; Kehlet, H. Safety aspects of preoperative high-dose glucocorticoid in primary total knee replacement. Br. J. Anaesth. 2017, 119, 267-275. [CrossRef] [PubMed]

38. Tang, H.; Li, H.; Zhang, S.; Wang, Y.; Qu, X.; Yue, B. Postoperative Complications Causing Readmission in 30 Days after Total Knee Arthroplasty: A Retrospective Nested Case-Control Study of Risk Factors Based on Propensity Score Matching. J. Knee Surg. 2019, 29, 29. [CrossRef] [PubMed]

39. Tay, K.S.; Cher, E.W.L.; Zhang, K.; Tan, S.B.; Howe, T.S.; Koh, J.S.B. Comorbidities Have a Greater Impact Than Age Alone in the Outcomes of Octogenarian Total Knee Arthroplasty. J. Arthroplast. 2017, 32, 3373-3378. [CrossRef]

40. Liao, K.M.; Lu, H.Y. Complications after total knee replacement in patients with chronic obstructive pulmonary disease: A nationwide case-control study. Medicine 2016, 95, e4835. [CrossRef]

41. Kuo, F.C.; Lin, P.C.; Lu, Y.D.; Lee, M.S.; Wang, J.W. Chronic Kidney Disease Is an Independent Risk Factor for Transfusion, Cardiovascular Complication, and Thirty-Day Readmission in Minimally Invasive Total Knee Arthroplasty. J. Arthroplast. 2017, 32, 1630-1634. [CrossRef] 
42. Ali, A.M.; Loeffler, M.D.; Aylin, P.; Bottle, A. Predictors of 30-Day Readmission After Total Knee Arthroplasty: Analysis of 566,323 Procedures in the United Kingdom. J. Arthroplast. 2019, 34, 242-248.e241. [CrossRef]

43. Miric, A.; Inacio, M.C.S.; Kelly, M.P.; Namba, R.S. Can Total Knee Arthroplasty Be Safely Performed Among Nonagenarians? An Evaluation of Morbidity and Mortality Within a Total Joint Replacement Registry. J. Arthroplast. 2014, 29, 1635-1638. [CrossRef]

44. Welsh, R.L.; Graham, J.E.; Karmarkar, A.M.; Leland, N.E.; Baillargeon, J.G.; Wild, D.L.; Ottenbacher, K.J. Effects of Postacute Settings on Readmission Rates and Reasons for Readmission Following Total Knee Arthroplasty. J. Am. Med. Dir. Assoc. 2017, 18, 367.e1-367.e10. [CrossRef]

45. Kim, S.C.; Jin, Y.; Lee, Y.C.; Lii, J.; Franklin, P.D.; Solomon, D.H.; Franklin, J.M.; Katz, J.N.; Desai, R.J. Association of Preoperative Opioid Use With Mortality and Short-term Safety Outcomes After Total Knee Replacement. JAMA Netw. Open 2019 , 2, e198061. [CrossRef]

46. Kurtz, S.M.; Lau, E.C.; Ong, K.L.; Adler, E.M.; Kolisek, F.R.; Manley, M.T. Which Hospital and Clinical Factors Drive 30- and 90-Day Readmission After TKA? J. Arthroplast. 2016, 31, 2099-2107. [CrossRef] [PubMed]

47. Anthony, C.A.; Peterson, R.A.; Sewell, D.K.; Polgreen, L.A.; Simmering, J.E.; Callaghan, J.J.; Polgreen, P.M. The Seasonal Variability of Surgical Site Infections in Knee and Hip Arthroplasty. J. Arthroplast. 2018, 33, 510-514.e511. [CrossRef] [PubMed]

48. Urish, K.L.; Qin, Y.; Li, B.Y.; Borza, T.; Sessine, M.; Kirk, P.; Hollenbeck, B.K.; Helm, J.E.; Lavieri, M.S.; Skolarus, T.A.; et al. Predictors and Cost of Readmission in Total Knee Arthroplasty. J. Arthroplast. 2018, 33, 2759-2763. [CrossRef] [PubMed]

49. Sodhi, N.; Mont, M.A.; Cleveland Clinic Orthopaedic, A. Does Patient Experience After a Total Knee Arthroplasty Predict Readmission? J. Arthroplast. 2019, 34, 2573-2579. [CrossRef] [PubMed]

50. Singh, J.A.; Kwoh, C.K.; Richardson, D.; Chen, W.; Ibrahim, S.A. Sex and surgical outcomes and mortality after primary total knee arthroplasty: A risk-adjusted analysis. Arthritis Care Res. 2013, 65, 1095-1102. [CrossRef] [PubMed]

51. Arroyo, N.S.; White, R.S.; Gaber-Baylis, L.K.; La, M.; Fisher, A.D.; Samaru, M. Racial/ethnic and socioeconomic disparities in total knee arthroplasty 30-and 90-day readmissions: A multi-payer and multistate analysis, 2007-2014. Popul. Health Manag. 2019, 22, 175-185. [CrossRef]

52. Bullock, D.P.; Sporer, S.M.; Shirreffs, T.G., Jr. Comparison of simultaneous bilateral with unilateral total knee arthroplasty in terms of perioperative complications. J. Bone Jt. Surg. Am. Vol. 2003, 85-A, 1981-1986. [CrossRef]

53. Charette, R.S.; Sloan, M.; DeAngelis, R.D.; Lee, G.C. Higher Rate of Early Revision Following Primary Total Knee Arthroplasty in Patients Under Age 55: A Cautionary Tale. J. Arthroplast. 2019. [CrossRef]

54. D'Apuzzo, M.; Westrich, G.; Hidaka, C.; Jung Pan, T.; Lyman, S. All-Cause Versus Complication-Specific Readmission Following Total Knee Arthroplasty. J. Bone Jt. Surg. Am. Vol. 2017, 99, 1093-1103. [CrossRef]

55. Keeney, J.A.; Nam, D.; Johnson, S.R.; Nunley, R.M.; Clohisy, J.C.; Barrack, R.L. Socioeconomically Disadvantaged CMS Beneficiaries Do Not Benefit From the Readmission Reduction Initiatives. J. Arthroplast. 2015, 30, 2082-2085. [CrossRef]

56. Ramos, N.L.; Karia, R.J.; Hutzler, L.H.; Brandt, A.M.; Slover, J.D.; Bosco, J.A. The effect of discharge disposition on 30-day readmission rates after total joint arthroplasty. J. Arthroplasty 2014, 29, 674-677. [CrossRef] [PubMed]

57. Ricciardi, B.F.; Oi, K.K.; Daines, S.B.; Lee, Y.Y.; Joseph, A.D.; Westrich, G.H. Patient and Perioperative Variables Affecting 30-Day Readmission for Surgical Complications After Hip and Knee Arthroplasties: A Matched Cohort Study. J. Arthroplast. 2017, 32, 1074-1079. [CrossRef] [PubMed]

58. Saucedo, J.M.; Marecek, G.S.; Wanke, T.R.; Lee, J.; Stulberg, S.D.; Puri, L. Understanding readmission after primary total hip and knee arthroplasty: who's at risk? J. Arthroplast. 2014, 29, 256-260. [CrossRef] [PubMed]

59. Schaeffer, J.F.; Scott, D.J.; Godin, J.A.; Attarian, D.E.; Wellman, S.S.; Mather, R.C., III. The association of ASA class on total knee and total hip arthroplasty readmission rates in an academic hospital. J. Arthroplast. 2015, 30, 723-727. [CrossRef]

60. Workman, K.K.; Angerett, N.; Lippe, R.; Shin, A.; King, S. Thirty-Day Unplanned Readmission after Total Knee Arthroplasty at a Teaching Community Hospital: Rates, Reasons, and Risk Factors. J. Knee Surg. 2019, 10, 10. [CrossRef]

61. Siracuse, B.L.; Ippolito, J.A.; Gibson, P.D.; Ohman-Strickland, P.A.; Beebe, K.S. A Preoperative Scale for Determining Surgical Readmission Risk After Total Knee Arthroplasty. J. Bone Jt. Surg. Am. Vol. 2017, 99, e112. [CrossRef]

62. Weick, J.; Bawa, H.; Dirschl, D.R.; Luu, H.H. Preoperative opioid use is associated with higher readmission and revision rates in total knee and total hip arthroplasty. J. Bone Jt. Surg. Am. Vol. 2018, 100, 1171-1176. [CrossRef]

63. Kheir, M.M.; Clement, R.C.; Derman, P.B.; Flynn, D.N.; Speck, R.M.; Levin, L.S.; Fleisher, L.A. Are there identifiable risk factors and causes associated with unplanned readmissions following total knee arthroplasty? J. Arthroplast. 2014, 29, $2192-2196$. [CrossRef]

64. Anderson, B.J.; Liu, M.; Cui, X.; Stevens, M.; Arensman, M.A. Adverse Outcomes Associated With Inpatient Administration of Beers List Medications Following Total Knee Replacement. Clin. Ther. 2020, 42, 592-604.e1. [CrossRef]

65. Mudumbai, S.C.; Chung, P.; Nguyen, N.; Harris, B.; Clark, J.D.; Wagner, T.H.; Giori, N.J.; Stafford, R.S.; Mariano, E.R. Perioperative Opioid Prescribing Patterns and Readmissions After Total Knee Arthroplasty in a National Cohort of Veterans Health Administration Patients. Pain Med. 2019, 21, 595-603. [CrossRef]

66. Abola, M.V.; Tanenbaum, J.E.; Bomberger, T.T.; Knapik, D.M.; Fitzgerald, S.J.; Wera, G.D. Preoperative Hyponatremia Is Associated with Reoperation and Prolonged Length of Hospital Stay following Total Knee Arthroplasty. J. Knee Surg. 2018, 4, 4. [CrossRef] [PubMed]

67. Alvi, H.M.; Mednick, R.E.; Krishnan, V.; Kwasny, M.J.; Beal, M.D.; Manning, D.W. The Effect of BMI on 30 Day Outcomes Following Total Joint Arthroplasty. J. Arthroplast. 2015, 30, 1113-1117. [CrossRef] [PubMed] 
68. Antoniak, D.T.; Benes, B.J.; Hartman, C.W.; Vokoun, C.W.; Samson, K.K.; Shiffermiller, J.F. Impact of Chronic Kidney Disease in Older Adults Undergoing Hip or Knee Arthroplasty: A Large Database Study. J. Arthroplast. 2020, 35, 1214-1221.e5. [CrossRef] [PubMed]

69. Belmont, P.J., Jr.; Goodman, G.P.; Rodriguez, M.; Bader, J.O.; Waterman, B.R.; Schoenfeld, A.J. Predictors of hospital readmission following revision total knee arthroplasty. Knee Surg. Sports Traumatol. Arthrosc. 2016, 24, 3329-3338. [CrossRef]

70. Bovonratwet, P.; Tyagi, V.; Ottesen, T.D.; Ondeck, N.T.; Rubin, L.E.; Grauer, J.N. Revision Total Knee Arthroplasty in Octogenarians: An Analysis of 957 Cases. J. Arthroplast. 2018, 33, 178-184. [CrossRef]

71. Bovonratwet, P.; Fu, M.C.; Tyagi, V.; Gu, A.; Sculco, P.K.; Grauer, J.N. Is Discharge Within a Day of Total Knee Arthroplasty Safe in the Octogenarian Population? J. Arthroplast. 2019, 34, 235-241. [CrossRef]

72. Bovonratwet, P.; Shen, T.S.; Ast, M.P.; Mayman, D.J.; Haas, S.B.; Su, E.P. Reasons and Risk Factors for 30-Day Readmission After Outpatient Total Knee Arthroplasty: A Review of 3015 Cases. J. Arthroplast. 2020, 35, 2451-2457. [CrossRef]

73. Courtney, P.M.; Boniello, A.J.; Della Valle, C.J.; Lee, G.C. Risk Adjustment Is Necessary in Value-based Outcomes Models for Infected TKA. Clin. Orthop. 2018, 476, 1940-1948. [CrossRef]

74. Curtis, G.L.; Chughtai, M.; Khlopas, A.; Newman, J.M.; Sultan, A.A.; Sodhi, N.; Barsoum, W.K.; Higuera, C.A.; Mont, M.A. Perioperative Outcomes and Short-Term Complications Following Total Knee Arthroplasty in Chronically, Immunosuppressed Patients. Surg. Technol. Int. 2018, 32, 263-269.

75. Curtis, G.; Hammad, A.; Anis, H.K.; Sodhi, N.; Ehiorobo, J.O.; Higuera, C.A.; Little, B.E.; Mont, M.A.; Darwiche, H.F. Preoperative Functional Status Predicts Increased Morbidity Following Total Knee Arthroplasty. Surg. Technol. Int. 2019, $34,1$.

76. George, J.; Piuzzi, N.S.; Ng, M.; Sodhi, N.; Khlopas, A.A.; Mont, M.A. Association Between Body Mass Index and Thirty-Day Complications After Total Knee Arthroplasty. J. Arthroplast. 2018, 33, 865-871. [CrossRef] [PubMed]

77. Gwam, C.U.; Sultan, A.; Rosas, S.; Sullivan, R.; Seem, M.; Ohanale, C.; Plate, J.F. Dialysis Is Not Associated with Increased Risk of Perioperative Complications in TKA Patients after Adjusting for Pertinent Confounders. J. Knee Surg. 2020, 33, 745-749. [CrossRef] [PubMed]

78. Hart, A.; Antoniou, J.; Brin, Y.S.; Huk, O.L.; Zukor, D.J.; Bergeron, S.G. Simultaneous Bilateral Versus Unilateral Total Knee Arthroplasty: A Comparison of 30-Day Readmission Rates and Major Complications. J. Arthroplast. 2016, 31, 31-35. [CrossRef] [PubMed]

79. Jauregui, J.J.; Cherian, J.J.; Pierce, T.P.; Elmallah, R.K.; Mont, M.A. Thirty-Day Readmission Rates Comparing Specific Cruciate Retaining and Posterior Stabilizing Knee Arthroplasties to the National Readmission Rates. J. Autom. Inf. Sci. 2015, 25, 307-311. [CrossRef]

80. Kester, B.S.; Minhas, S.V.; Vigdorchik, J.M.; Schwarzkopf, R. Total Knee Arthroplasty for Posttraumatic Osteoarthritis: Is it Time for a New Classification? J. Arthroplast. 2016, 31, 1649-1653.e1641. [CrossRef]

81. Lehtonen, E.J.; Hess, M.C.; McGwin, G., Jr.; Shah, A.; Godoy-Santos, A.L.; Naranje, S. Risk Factors for Early Hospital Readmission Following Total Knee Arthroplasty. Acta Ortop 2018, 26, 309-313. [CrossRef]

82. Lovecchio, F.; Beal, M.; Kwasny, M.; Manning, D. Do patients with insulin-dependent and noninsulin-dependent diabetes have different risks for complications after arthroplasty? Clin. Orthop. Relat. Res. 2014, 472, 3570-3575. [CrossRef]

83. Nowak, L.L.; Schemitsch, E.H. Same-day and delayed hospital discharge are associated with worse outcomes following total knee arthroplasty. Bone Jt. J. 2019, 101-B, 70-76. [CrossRef]

84. Ottesen, T.D.; Zogg, C.K.; Haynes, M.S.; Malpani, R.; Bellamkonda, K.S.; Grauer, J.N. Dialysis Patients Undergoing Total Knee Arthroplasty Have Significantly Increased Odds of Perioperative Adverse Events Independent of Demographic and Comorbidity Factors. J. Arthroplast. 2018, 33, 2827-2834. [CrossRef]

85. Patel, A.P.; Gronbeck, C.; Chambers, M.; Harrington, M.A.; Halawi, M.J. Gender and Total Joint Arthroplasty: Variable Outcomes by Procedure Type. Arthroplast. Today 2020, 6, 517-520. [CrossRef]

86. Patterson, J.T.; Tillinghast, K.; Ward, D. Dialysis Dependence Predicts Complications, Intensive Care Unit Care, Length of Stay, and Skilled Nursing Needs in Elective Primary Total Knee and Hip Arthroplasty. J. Arthroplast. 2018, 33, 2263-2267. [CrossRef] [PubMed]

87. Robinson, J.; Shin, J.I.; Dowdell, J.E.; Moucha, C.S.; Chen, D.D. Impact of Gender on 30-Day Complications After Primary Total Joint Arthroplasty. J. Arthroplast. 2017, 32, 2370-2374. [CrossRef] [PubMed]

88. Roth, A.; Khlopas, A.; George, J.; Churchill, J.L.; Molloy, R.; Mont, M.A.; Piuzzi, N.S.; Higuera, C.A. The Effect of Body Mass Index on 30-day Complications After Revision Total Hip and Knee Arthroplasty. J. Arthroplast. 2019. [CrossRef] [PubMed]

89. Rudasill, S.E.; Liu, J.; Kamath, A.F. Revisiting the International Normalized Ratio (INR) Threshold for Complications in Primary Total Knee Arthroplasty: An Analysis of 21,239 Cases. J. Bone Jt. Surg. Am. Vol. 2019, 101, 514-522. [CrossRef]

90. Runner, R.P.; Bellamy, J.L.; Vu, C.C.L.; Erens, G.A.; Schenker, M.L.; Guild, G.N., 3rd. Modified Frailty Index Is an Effective Risk Assessment Tool in Primary Total Knee Arthroplasty. J. Arthroplast. 2017, 32, S177-S182. [CrossRef]

91. Sloan, M.; Sheth, N.P.; Nelson, C.L. Obesity and hypoalbuminaemia are independent risk factors for readmission and reoperation following primary total knee arthroplasty. Bone Jt. J. 2020, 102-B, 31-35. [CrossRef]

92. Sodhi, N.; Anis, H.K.; Coste, M.; Ehiorobo, J.O.; Chee, A.; Freund, B.; Sultan, A.A.; Moskal, J.T.; Scuderi, G.R.; Mont, M.A. A Nationwide Analysis of Preoperative Planning on Operative Times and Postoperative Complications in Total Knee Arthroplasty. J. Knee Surg. 2019, 32, 1040-1045. [CrossRef] 
93. Suleiman, L.I.; Edelstein, A.I.; Thompson, R.M.; Alvi, H.M.; Kwasny, M.J.; Manning, D.W. Perioperative Outcomes Following Unilateral Versus Bilateral Total Knee Arthroplasty. J. Arthroplast. 2015, 30, 1927-1930. [CrossRef]

94. Sutton, J.C.; Antoniou, J.; Epure, L.M.; Huk, O.L.; Zukor, D.J.; Bergeron, S.G. Hospital Discharge within 2 Days Following Total Hip or Knee Arthroplasty Does Not Increase Major-Complication and Readmission Rates. J. Bone Jt. Surg. Am. Vol. 2016, 98, 1419-1428. [CrossRef]

95. Webb, M.L.; Golinvaux, N.S.; Ibe, I.K.; Bovonratwet, P.; Ellman, M.S.; Grauer, J.N. Comparison of Perioperative Adverse Event Rates After Total Knee Arthroplasty in Patients With Diabetes: Insulin Dependence Makes a Difference. J. Arthroplast. 2017, 32, 2947-2951. [CrossRef]

96. Yohe, N.; Funk, A.; Ciminero, M.; Erez, O.; Saleh, A. Complications and Readmissions After Total Knee Replacement in Octogenarians and Nonagenarians. Geriatr 2018, 9, 2151459318804113. [CrossRef] [PubMed]

97. Zusmanovich, M.; Kester, B.S.; Schwarzkopf, R. Postoperative Complications of Total Joint Arthroplasty in Obese Patients Stratified by BMI. J. Arthroplast. 2018, 33, 856-864. [CrossRef] [PubMed]

98. Sterne, J.A.C.; Sutton, A.J.; Ioannidis, J.P.A.; Terrin, N.; Jones, D.R.; Lau, J.; Carpenter, J.; Rücker, G.; Harbord, R.M.; Schmid, C.H.; et al. Recommendations for examining and interpreting funnel plot asymmetry in meta-analyses of randomised controlled trials. BMJ 2011, 343, d4002. [CrossRef] [PubMed]

99. World Health Organization. Obesity: Preventing and Managing the Global Epidemic; World Health Organization: Geneva, Switzerland, 2000.

100. Brophy, H.F. Publications of the United Nations Statistical Office. Gov. Publ. Rev. 1982, 9, 175-184. [CrossRef]

101. Shiloach, M.; Frencher, S.K., Jr.; Steeger, J.E.; Rowell, K.S.; Bartzokis, K.; Tomeh, M.G.; Richards, K.E.; Ko, C.Y.; Hall, B.L. Toward robust information: Data quality and inter-rater reliability in the American College of Surgeons National Surgical Quality Improvement Program. J. Am. Coll. Surg. 2010, 210, 6-16. [CrossRef]

102. Kristensen, S.R.; Bech, M.; Quentin, W. A roadmap for comparing readmission policies with application to Denmark, England, Germany and the United States. Health Policy 2015, 119, 264-273. [CrossRef] 